\title{
Validation of a new technique to freezing and embedding tissue in Mohs surgery, using an animal model ${ }^{1}$
}

\author{
Frederico Hassin Sanchez ${ }^{I}$, Joaquim Ribeiro Filho" ${ }^{\text {II }}$ Asdrubal Cesar Russo ${ }^{\text {III }}$ \\ DOI: http://dx.doi.org/10.1590/S0102-865020160080000006
}

${ }^{\mathrm{I}}$ Fellow Master degree, Postgraduate Program in Surgical Sciences, Medical School, Universidade Federal do Rio de Janeiro (UFRJ), Brazil. Conception and design of the study, acquisition of data, manuscript writing.

IIPhD, Associate Professor, Postgraduate Program in Surgical Sciences, Medical School, UFRJ, Rio de Janeiro-RJ, Brazil. Conception and design of the study, critical revision, final approval of the version to be published.

IIIFull Professor, Graduation Program in medicine, Medical School, Universidade Federal Fronteira Sul (UFFS), Chapecó-SC, Brazil. Acquisition of data.

\section{ABSTRACT}

PURPOSE: To validate the innovative Dry Ice method, comparing it with two standard methods currently used for tissue processing in Mohs surgery, the Heat Sink method and the Miami Special.

METHODS: Forty eight samples of pigs kin with the standard beveled Mohs technique were used, and randomly allocated into six groups. Each group was processed with one of the 3 methods and evaluated for: The freezing time, the depth required to cut into the block to obtain a complete section, and the quality of histological slides analyzed with a image software. The statistical analysis was performed with the software $\mathrm{SAS}^{\circledR}$ System. The inferential analysis was made by one-way ANOVA.

RESULTS: The Miami Special showed a processing time significantly shorter than Dry Ice method and Heat Sink method. There was no significant difference in the depth required to cut into the blocks, and area of surgical margins visualized.

CONCLUSION: The Dry Ice method was as efficient as the other two methods currently used in Mohs surgery, considering the individual advantages and disadvantages of each method.

Key words: Mohs Surgery. Skin Neoplasms. Dermatology. Equipment and Supplies. Inventions. Swine. 


\section{Introduction}

The Mohs micrographic surgery is a gold standard procedure to treat several types of skin cancer ${ }^{1}$. It is a meticulous procedure that includes a number of technical steps, from the tumor removal until the tissue preparation, mapping the surgical margins and the microscopic analysis.

The tissue processing performed in Mohs surgery distinguishes from the conventional histological techniques. In conventional standard procedures, the tissues are frozen, and cut, like a loaf of bread (bread-loaf technique) or variations thereof, allowing only a small sampling of all surgical margins to be analyzed, causing false negatives, and increasing the chance of tumor recorrence ${ }^{2,3}$. Consequently in the conventional histologic techniques, generally 2 to $4 \mathrm{~mm}$ of tissue is left unexamined at each interval, and less than $1 \%$ of the excised margin is analysed ${ }^{2-4}$. In contrast, during Mohs surgery, the tissue is removed with a specific angulation of the scalpel, allowing flattening of the lateral and deep surgical margins in the same plane. This sectioning of flat tissue, allows the confection of histological slides containing all surgical margins in the same section, allowing the analysis of $100 \%$ of all surgical margins $s^{2,5,6}$.

The great rate of efficacy of Mohs surgery is attributed to the ability to view all surgical margins in a single en face section ${ }^{5,6}$. The high quality frozen sections are fundamental to allow the visualization of the peripheral and deep margins in the same plane ${ }^{7}$.

Each successive step of the surgery demands time, and the patient needs to wait for the tissue processing and the microscopic analysis. When large tumors are removed and multiple samples need to be processed, a long waiting time can be necessary, and can increase the morbidity, the staff fatigue and technical errors ${ }^{8}$. Many innovative techniques have been developed to improve efficiency and speed of the tissue processing in Mohs surgery, keeping the high quality of histological slides ${ }^{8,9}$.

A survey of Mohs surgeons published in 2003 showed a great variety of the devices and techniques used to flatten, to freeze, and to prepare the tissues in Mohs surgery, among them, the heat extractor flattening in the cryostat (22.6\%), relaxing incisions $(16.1 \%)$, heat extractor and relaxing incisions (14.2\%), freezing on a glass slide $(10.3 \%)$, relaxing incision and glass slide $(9.4 \%)$, and the Miami Special technique (3.9\%) are the most commonly used $^{10}$. Other less used devices, such as the Davidson Cryocup ${ }^{\mathrm{TM}}$ (Bradley Products Inc., Bloomington, MN), CryoHist ${ }^{\mathrm{TM}}$ (Cryo Histology, Inc., Shawnee, KS), the Cryo-Embedder ${ }^{\mathrm{TM}}$ ( Salt Lake City, UT), the face-down cryoembedding of tissue using stainless stell embedding wells, and the use of dry ice have been described to optimize the tissue preparation process ${ }^{8,9,11,12}$.

One of the most popular techniques used to flatten and freeze specimens is the heat extractor method, used for flattening and freezing the tissues inside the cryostat ${ }^{12,13}$. However, the warm weather and high temperatures, increase the thermal exchange between the cryostat chamber and the environment, and it can slow down the process, especially when many samples need to be processed ${ }^{7,12}$. In an attempt to minimize this problem, some surgeons have used over 30 years the Miami Special method, that uses a specific device to mechanical flattening with liquid nitrogen ${ }^{7}$. In 2014 a promising process for freezing and embedding tissue with dry ice was described, which did not require the use of cryostat or liquid nitrogen to freeze the tissue ${ }^{12}$. However despite the apparent cost-effectiveness advantages, there is a lack of comparative studies.

The purpose of this article is to compare the innovative dry ice technique ${ }^{12}$ with two standard methods currently used for tissue processing in Mohs surgery, the Heat Sink method ${ }^{5,13}$ and the Miami Special ${ }^{7}$, using an animal model.

\section{Methods}

The research was approved by the Ethics Committee for the use on animal experimentation of the Universidade Federal do Rio de Janeiro (UFRJ) (150-13).

Porcine skin from two newly slaughtered animals (Sus scrofa domestica) was used and donated by an official inspected slaughterhouse (Cooperativa Central Aurora Alimentos, Chapecó$\mathrm{SC})$.

A large area of pig belly skin was excised from the two animals, and immediately cooled until the use in the same day. The same author marked and excised circular samples $1.0 \mathrm{~cm}$ in diameter from the pig belly skin, using the standard beveled Mohs technique. Forty eight specimens were randomly allocated into six groups of samples. The group 1, group 2 and group 3, were composed of 10 circular samples each one. A cut in the middle of the circular samples was performed to promote the relaxation of the edges ${ }^{14,15}$. The remaining 18 samples were randomly allocated into three groups of 06 samples each one. The samples of the groups 4 , group 5, and group 6, were bisected and processed as two separate pieces, resulting in 12 semi-circular samples in each group (Figure 1). The processing as two parts, is a laboratory technique commonly used in Mohs surgery, especially used when preparing a thicker skin sample, to facilitate the flattening and to allow better assessment of surgical margins (Figure 2) ${ }^{5,14}$. Each group of the not divided samples, and each group of bisected 
samples were processed as one of the three methods of freezing and tissue processing (Table 1).

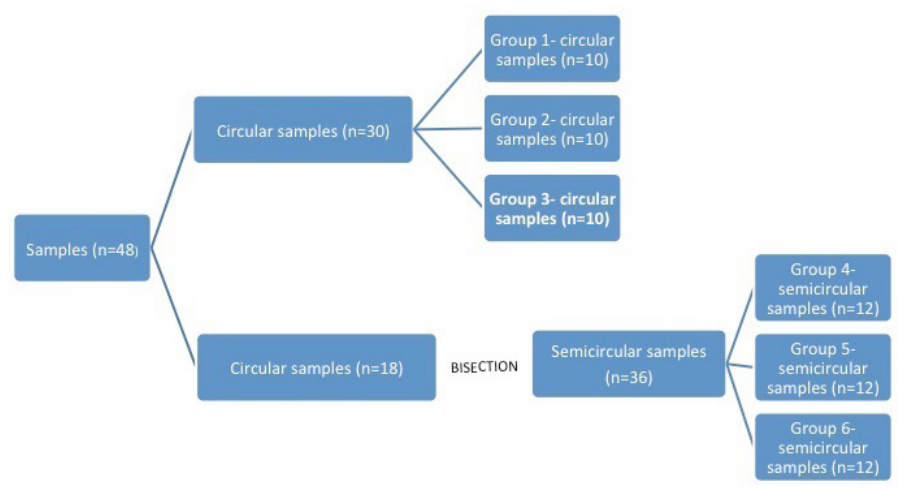

FIGURE 1 - Flowchart of the arrangement of samples in each group.

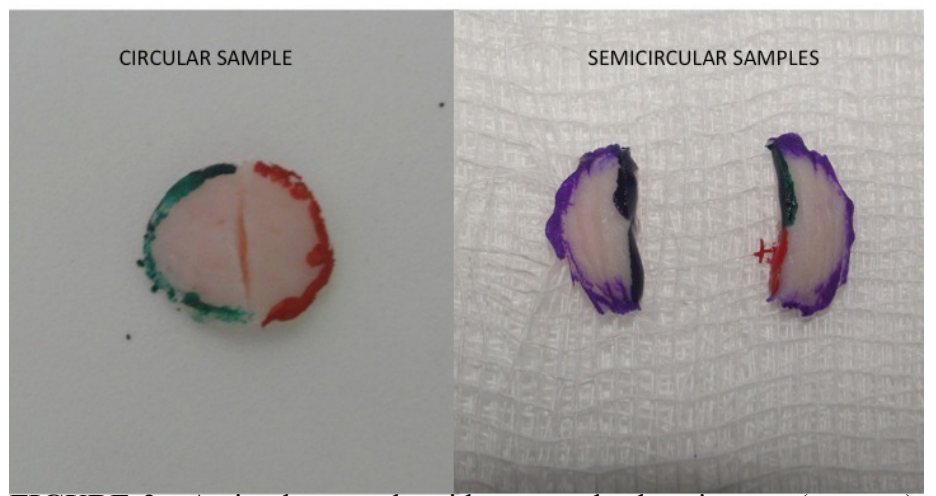

FIGURE 2 - A circular sample with a central relaxation cut (pacman), and a bisected semicircular sample.

TABLE 1 - Characteristic of the groups.

\begin{tabular}{|c|c|c|c|}
\hline Groups & $\begin{array}{l}\text { Status of } \\
\text { Samples }\end{array}$ & $\begin{array}{c}\text { Number of } \\
\text { samples }(n)\end{array}$ & $\begin{array}{l}\text { Method of } \\
\text { processing }\end{array}$ \\
\hline Group 1 & Circular & 10 & Heat sink method \\
\hline Group 2 & Circular & 10 & $\begin{array}{c}\text { Miami Special } \\
\text { method }\end{array}$ \\
\hline Group 3 & Circular & 10 & Dry Ice method \\
\hline Group 4 & Semicircular & 12 & Heat Sink method \\
\hline Group 5 & Semicircular & 12 & $\begin{array}{c}\text { Miami Special } \\
\text { method }\end{array}$ \\
\hline Group 6 & Semicircular & 12 & Dry Ice method \\
\hline
\end{tabular}

The margins of the all specimens were inked like standard Mohs technique. Some authors recommend the application of en face ink to detect and define embedding errors in Mohs specimens ${ }^{16,17}$. The standard ink was used in the edge of the all samples, for easy viewing to the end point of sectioning by histotechnician.
To eliminate technician variability in the results, a single and experienced histology technician with proficiency in the three methods worked on the project. For each method the same embedding medium was used in the preparation of all samples: The Optimal Cutting Temperature Compound (OCT) (Tissue$\mathrm{Tek}^{\circledR}$, Sakura Finetek USA, inc., Torrence, CA).

The tissue processing with each technique were the following:

\section{Heat extractor method (Heat Sink technique)}

In the heat extractor method, the sample is placed with surgical margin side down on a chilled heat extractor and the epidermal edge is gently laid flat on the cold heat sink surface to allow flattening the deep and peripheral margin in a single plane. The OCT is applied on the specimen on the heat extractor, covering it entirely and left to freeze until it gets an opaque appearance. OCT is applied to a cutting chuck to freeze inside the cryostat. The heat extractor with the tissue is immediately inverted onto the chuck containing OCT, and they freeze together as a sandwich. After freezing, the heat extractor is pulled away from the chuck containing the tissue. An additional drop of the OCT is applied on the frozen block surface to fill clefts at the tissue and then the heat extractor is pressed against to the block surface for few seconds, leaving the cutting chuck prepared for sectioning ${ }^{5}$ (Figure 3 ).
1)

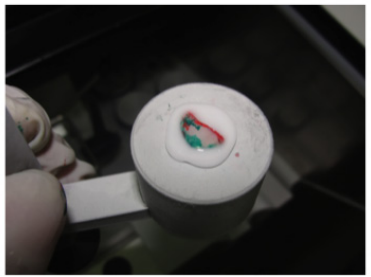

3)

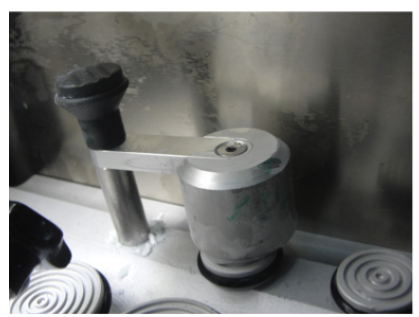

2)

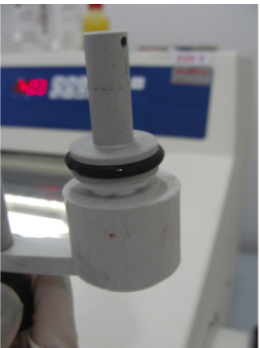

4)

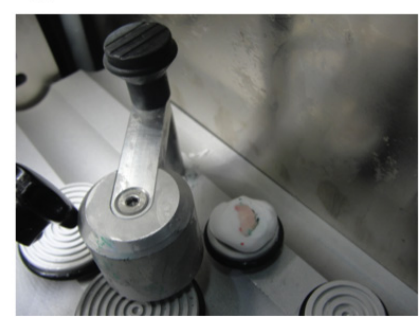

FIGURE 3 - Heat Sink method. 1) The sample placed with surgical margin side down on a chilled heat extractor, and the OCT is applied on the specimen on the heat extractor, covering it entirely. 2) The heat extractor with the tissue is immediately inverted onto the chuck containing OCT, and they freeze together as a sandwich. 3 and $\mathbf{4}$ ) The heat extractor is pressed against to the block surface for few seconds, leaving the cutting chuck prepared for sectioning. 


\section{Liquid nitrogen method (Miami Special technique)}

An adapted obstetric clamp has been used at the University of Miami for over 30 years, to facilitate the embedding tissue process in the hot and humid environment of Florida ${ }^{7}$ (Figure 4). The device is designed to hold a chuck and a portion of tissue together with parallel faces while being dipped in liquid nitrogen to rapidly freeze with tissue and OCT thus forming a block. A small amount of embedding medium is placed over the chuck from the cryostat and dipped for few seconds in liquid nitrogen until it acquires a gray translucent appearance ${ }^{8}$. The chuck is placed into the chuck holder of the Miami Special clamp. On the opposite side of the device, the tissue sample is gently placed and flattened on the plane surface the Miami Special clamp with deep and peripheral margins in the same flat plane. The Miami Special device is closed and the chuck is clamped into fixed position like a common hemostat, allowing the sandwiched tissue and chuck to be dipped into liquid nitrogen to freeze together ${ }^{18}$. The device is opened and an additional drop of OCT is applied on the tissue surface to fill any clefts, and then it is closed again to press the tissue surface against to the device flat surface and dipped for few seconds to complete the freezing process ${ }^{18}$. The chuck with the embedding tissue is removed from the device ready for sectioning (Figure 4).
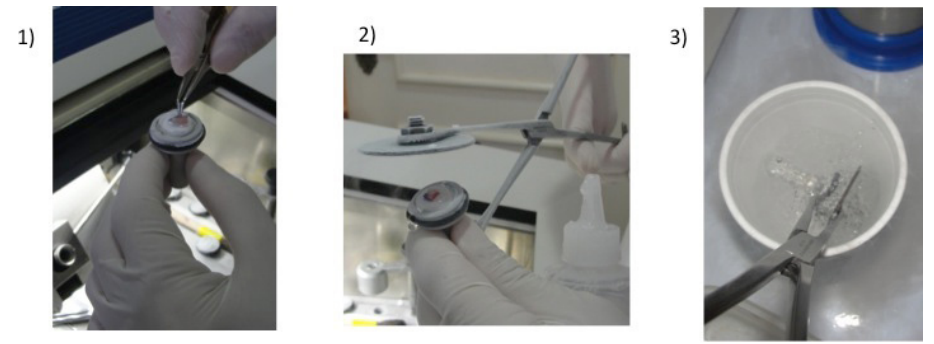

4)

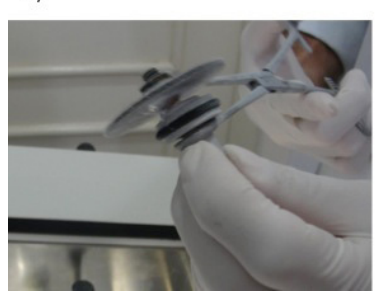

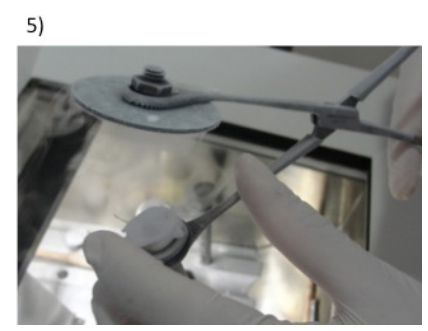

FIGURE 4 - Miami Special method. 1) The sample is gently placed on the chuck. 2) The chuck is placed into the chuck holder of the Miami Special clamp. 3) The sandwiched tissue and chuck are dipped into liquid nitrogen to freeze together. 4) The device is opened and an additional drop of OCT is applied on the tissue surface to fill any clefts, and then it is closed again to press the tissue surface against to the device flat surface and dipped for few seconds to complete the freezing. 5) The chuck with the embedding tissue is removed from the device ready for sectioning.

\section{Dry ice method (Rio de Janeiro technique)}

This technique was developed to accelerate the embedding tissue process in the hot climate of Rio de Janeiro (Brazil) and uses an aluminum box containing 8-12 dry ice cubes, with a thermal insulation surrounding the side and bottom surfaces of the box (polyurethane support) ${ }^{12}$. The flat upper surface of the box, which we called the external freezing bar (EFB) works just like the internal freezing bar of the cryostat, with the advantage that it enables to handle the samples outside of the cryostat and allow to process several samples simultaneously. The specimen is placed on the surface of EFB with lower surgical margin facing down, and the peripheral margins are flattened on the bar surface. Gentle pressure with a finger is applied to the center of the sample to ensure that the deep and peripheral margins are in contact with the frozen surface. The OCT medium is applied directly over the specimen, and an additional OCT is applied on the chuck from the cryostat. The embedding medium on the metal surface and the chuck must be only partially frozen when sandwiched together so they will freeze together in a solid block. After few seconds the freezing block is separated from the EFB and a small additional portion of OCT is applied to the surface of the embedding tissue on the prepared cutting chuck and pressed against the EFB for approximately 10 seconds to flatten the surface. The prepared chuck is ready to be transferred to the cryostat for sectioning $\left(\right.$ Figure 5) ${ }^{12}$.

a)

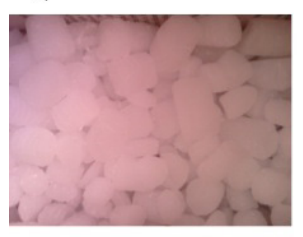

d)

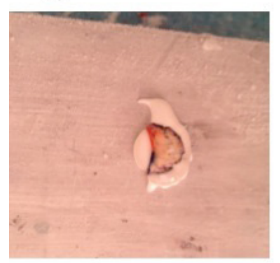

FIGURE 5 - a) Dry ice inside the device; b) Dry ice inside the device; c) The semicircular sample flattened on the plane surface of the device; d) The sample freezing with the OCT; e) Chucks against the freezing samples; f) The cutting chuck ready to sectioning, with the deep and lateral margins of the sample in the same flat plane. 
For each group, the time of the tissue processing and the mounting of the blocks was recorded, from the freezing, until ready for sectioning, inside the cryostat. Because all specimens were treated the same after this, the end point was the samples ready for sectioning inside the cryostat.

In all samples, sections every 5 microns $(\mu \mathrm{m})$ were performed, until it was possible to view the depth margin, and epidermal margin in a same plane. The depth required to cut into the block to obtain a complete section was recorded in microns.

These samples were stained with hematoxylin and eosin, and then mounted with glass coverslips standardly.

The same investigator evaluated only one slide of each sample, with the first complete section. Although the optimal session is defined by the complete representation of the epidermis, dermis and subcutaneous, many authors consider as a most important quality criterion of the slides, the visualization of more than $90 \%$ of epidermal margins ${ }^{5,8,11}$. The sections were microscopically analyzed by an experimented surgeon, and the complete sections were defined as having at least $90 \%$ of the epidermis and a complete representation of dermis and fat tissue, and the visualization of the inke in all surgical margins. It was the end point to stop the sectioning of the block. In a second moment, the slides were scanned using a slide scanner (Aperio ScanScope slide scanner ${ }^{\mathrm{TM}}$ - Leica Biosystems) and the software ImageJ (Rasband, W.S., ImageJ, U. S. National Institutes of Health, Bethesda, Maryland, USA.) was used to estimate the visualization of the margin in each slide (Figures 6 and 7).
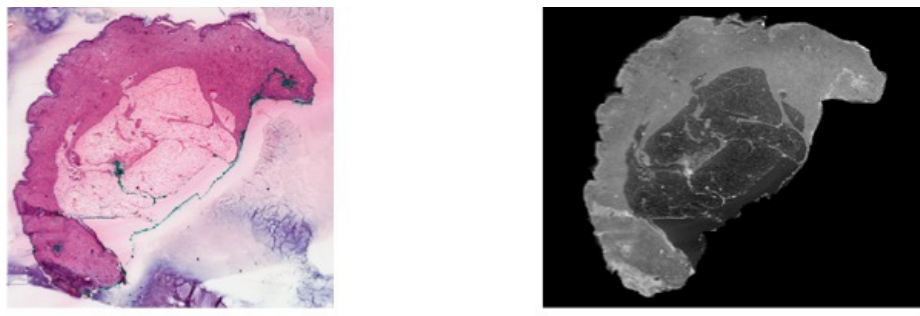

c)

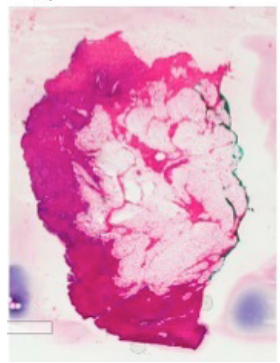

d)

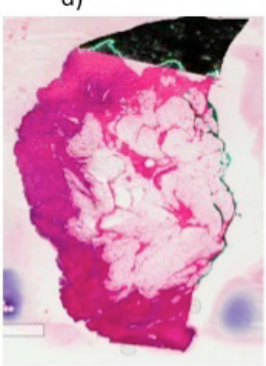

FIGURE 6 - a) The scanned histological slide, with entire deep and peripheral margin visualized; b) The area of the histological slide analyzed by the ImageJ software; c) A histological sample with lost of part of surgical margins; d) The estimate visualized area of the sample analyzed by the ImageJ software.

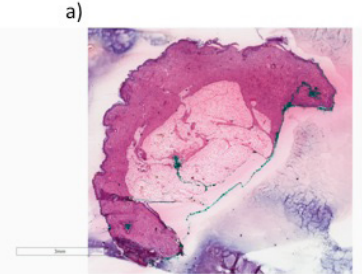

b)

c)
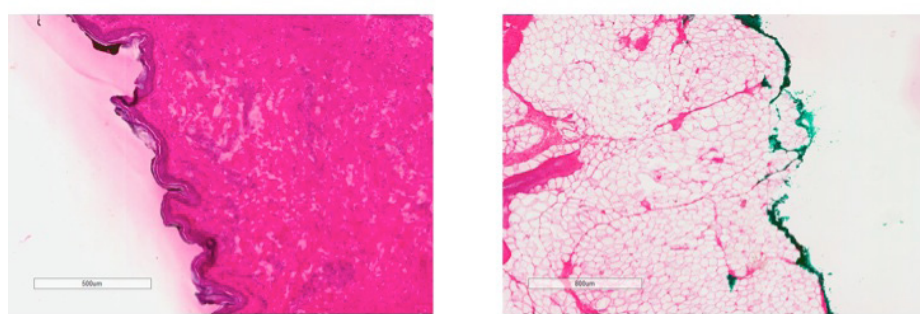

FIGURE 7 - a) The entire semicircular sample; b) A detail of the peripheral margin with the epidermis; c) A detail of the deep margin delimited by the green ink.

\section{Statistical analysis}

Statistical analysis was performed with the statistical software SAS ${ }^{\circledR}$ System, version 6.11 (SAS Institute, Inc., Cary, North Carolina). The inferential analysis was made by the statistical method one-way ANOVA, and the multiple comparison test of Tukey. The normality hypothesis was not rejected according to the Shapiro-Wilks test. The significance of determining the level adopted was 5\%. The analytical design was defined by one-way ANOVA for each type of sample, rather than two-way ANOVA, with the main objective to analyze the influence of the each method separately for each sample type, circular (Groups 1, 2 and 3) or semi-circular (Groups 4, 5 and 6).

The descriptive analysis presented in tables, and the observed data were expressed as mean and standard deviation in each group. Illustrative graphics were constructed expressed by the mean, and $95 \%$ confidence interval.

\section{Results}

The Tables 2 and 3 show the mean and standard deviation (SD) of the processing time, the depth of sectioning into the block, and the percentage of visualized margins, according to freezing methods, and the corresponding descriptive level ( $p$ value) of the statistic test, of the circular samples and of the bisected semicircular samples, respectively. 
TABLE 2 - Result of the processing time, depth of the block sectioning, and margins visualized, in each group with circular samples.

\begin{tabular}{|c|c|c|c|c|c|c|}
\hline \multirow[t]{2}{*}{ Object of analysis } & Group / Method & mean & \pm & SD & p value ${ }^{a}$ & $\neq$ significant $^{b}$ \\
\hline & Group 1 (Heat Sink) & 168.8 & \pm & 18.3 & & Group $1 \neq$ Group 2 \\
\hline \multirow[t]{2}{*}{ Processing Time (seg) } & Group 2 (Liquid Nitrogen) & 73.1 & \pm & 7.8 & $<0.0001$ & Group $1 \neq$ Group 3 \\
\hline & Group 3 (Dry Ice) & 124.2 & \pm & 7.9 & & Group $2 \neq$ Group 3 \\
\hline \multirow{3}{*}{$\begin{array}{l}\text { Depth of the block } \\
\text { sectioning }(\mu \mathrm{m})\end{array}$} & Group 1 (Heat Sink) & 525.0 & \pm & 94.3 & & \\
\hline & Group 2 (Liquid Nitrogen) & 622.5 & \pm & 138.5 & 0.025 & Group $2 \neq$ Group 3 \\
\hline & Group 3 (Dry Ice) & 484,5 & \pm & 85,5 & & \\
\hline \multirow{3}{*}{ Margins Visualized (\%) } & Group 1 (Heat Sink) & 94.0 & \pm & 1.8 & & \\
\hline & Group 2 (Liquid Nitrogen) & 93.7 & \pm & 2.2 & 0.89 & \\
\hline & Group 3 (Dry Ice) & 94.1 & \pm & 1.8 & & \\
\hline
\end{tabular}

TABLE 3 - Result of the processing time, depth of the block sectioning, and margins visualized, in each group with semicircular samples.

\begin{tabular}{|c|c|c|c|c|c|c|}
\hline Object of Analysis & Group (Method) & mean & \pm & $\mathrm{SD}$ & p value ${ }^{a}$ & $\neq$ significant $^{b}$ \\
\hline \multirow{3}{*}{ Processing Time (sec) } & Group 3 (Heat Sink) & 129.1 & \pm & 18.2 & \multirow{3}{*}{$<0.0001$} & Group $3 \neq$ Group 4 \\
\hline & Group 4 (Liquid Nitrogen) & 71.3 & \pm & 6.0 & & Group $3 \neq$ Group 5 \\
\hline & Group 5 (Dry Ice) & 115.3 & \pm & 12.4 & & Group $4 \neq$ Group 5 \\
\hline \multirow{3}{*}{$\begin{array}{l}\text { Depth of the Block } \\
\text { Sectioning }(\mu \mathrm{m})\end{array}$} & Group 3 (Heat Sink) & 339.6 & \pm & 45.1 & \multirow{3}{*}{0.52} & \\
\hline & Group 4 (Liquid Nitrogen) & 364.6 & \pm & 58.8 & & \\
\hline & Group 5 (Dry Ice) & 352.1 & \pm & 54.2 & & \\
\hline \multirow{3}{*}{ Margins Visualized (\%) } & Group 3 (Heat Sink) & 95.6 & \pm & 2.0 & \multirow{3}{*}{0.93} & \\
\hline & Group 4 (Liquid Nitrogen) & 96.0 & \pm & 2.9 & & \\
\hline & Group 5 (Dry Ice) & 95.9 & \pm & 2.1 & & \\
\hline
\end{tabular}

For the groups containing circular samples (Groups 1, 2 and 3), according to one-way ANOVA analysis, there was a significant difference in processing time $(p<0.0001)$, and also in the depth required to the sectioning of the blocks $(p=0.025)$ between the groups. There was not a significant difference in the visualization of the surgical margins between the groups ( $\mathrm{p}=$ $0.89)$.

Applying the Tukey test, it was found that the group 2, which was used liquid nitrogen, showed a processing time significantly shorter than groups 1 (Heat Sink) and group 3 (dry ice). The group 3 (dry ice), showed a processing time significantly shorter than group 1 (Heat Sink). So the liquid nitrogen was more efficient to freeze the blocks, followed by Dry Ice and Heat Sink methods, respectively.

The group 2, processed with the Miami Special technique, required significantly more sectioning of the blocks than the group 3, processed with Rio de Janeiro technique. There was no significant difference between the group 1 and 2, and between the groups 1 and 3 .

For the groups containing semi-circular bisected samples (Groups 4, 5 and 6), according to ANOVA for one factor, there was a significant difference in the processing time $(\mathrm{p}<0.0001)$. There was no significant difference in the depth required to cut into the blocks, between the groups $(p=0.52)$. There was no difference in the area of surgical margins visualized with each method ( $p=$ $0.93)$. 
Applying the Tukey test to the groups with semi-circular samples, the group 5 had a processing time significantly shorter than the groups 5 and 6 . The group 6 showed a tissue processing time significantly shorter than group 4 .

The Figures 8, 9 and 10 show the mean values, considering a $95 \%$ confidence intervals for the three analyzed factors: The tissue processing time in seconds (sec), the depth required to cut into the block to obtain a complete section in microns $(\mu \mathrm{m})$, and the percentage area of the surgical margin visualized (\%), respectively in each group.

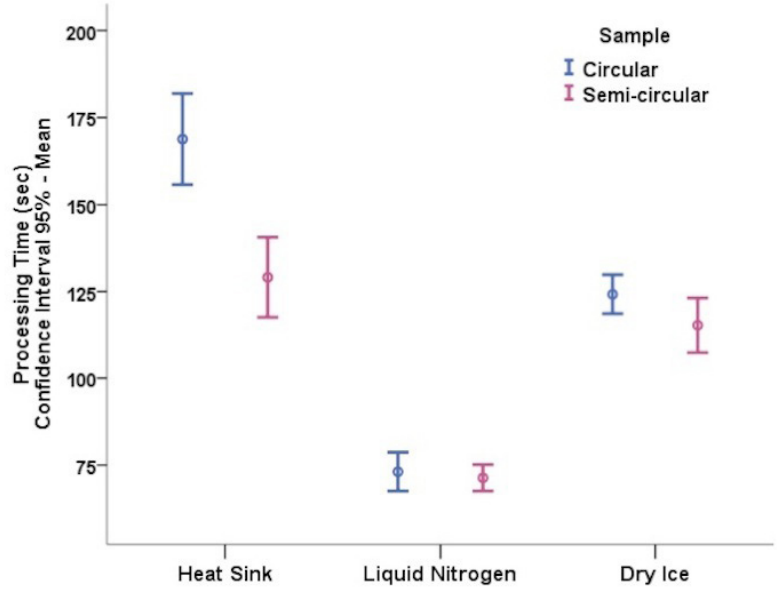

FIGURE 8 - Graphic with tissue processing time in each group.

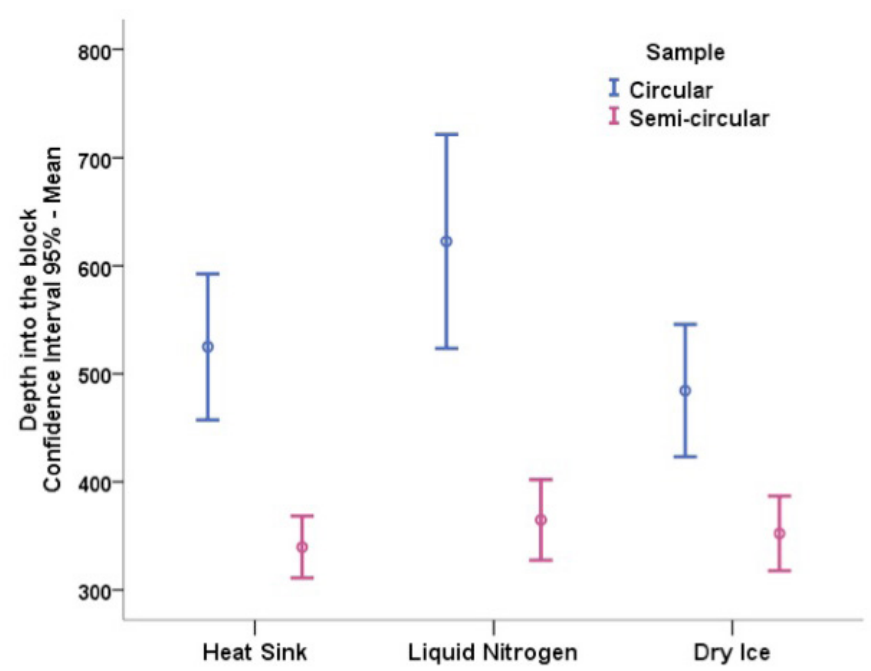

FIGURE 9 - The depth required to achieve the optimal section to cut the block.

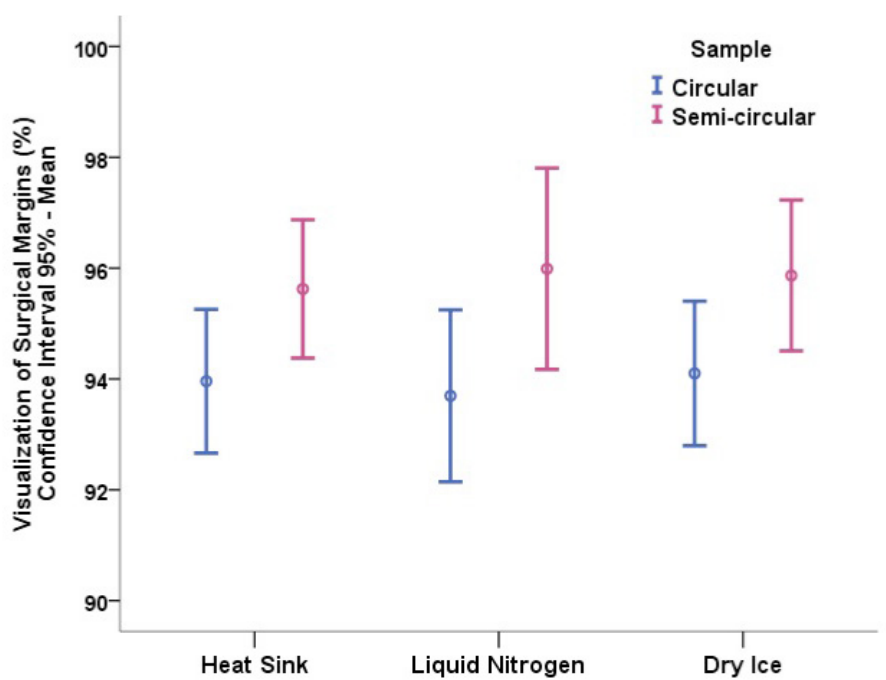

FIGURE 10 - Graphic of the percentage of the visualized area of the samples in each group.

\section{Discussion}

The main limitation of this study was the use of normal pigskin, disallowing the evaluation of the ability to detect tumor with each method (capacity to see the tumor on histological slides).

Normally the samples of up to one centimeter in diameter are possible to process as a single piece. A central cut to relax the edges known as pacman technique is usually enough to achieve an optimal flattening ${ }^{18,19}$.

Sometimes, due to the large thickness of the dermis, the samples need to be divided in halves, and embedding as two separate pieces, to allow adequate flattening. In this article we evaluated the freezing time of the bisected semi-circular samples separately. It is expected that the time to process two separate parts is multiplied by two $^{5}$. This increases the time spent in tissue processing compared to a processing of the single circular sample ${ }^{5,19}$ (Figure 8). However, the blocks with separate parts needed less thinning to achieve optimal section (Figure 9), because of this it is possible that the time lost in the inclusion of two separate samples is offset by the less time spent in the roughing of each block, although this has not been evaluated in this article ${ }^{5}$.

Each method used in this study has unique features, advantages and disadvantages.

The Miami Special was the faster method to embedding specimens when compared with the others, considering all types of samples. Considering the circular samples, the Miami Special 
technique required more thinning of the blocks to achieve optimal section. There were no statistical differences when thinning blocks with bisected semi-circular samples in the three methods.

The analysis showed that the Rio de Janeiro technique freezed the blocks faster than Heat sink method, however it was slower than the Miami Special technique in both types of samples. When circular samples were used, the technique of Rio de Janeiro needed less thinning of the blocks to achieve optimum cutting section. This shows that although the Miami Special technique has frozen samples faster, the technique of Rio de Janeiro requires less thinnig of the blocks, optimizing the time at this stage and can compensate the total tissue processing time. There was no difference when bisected semi-circular samples were used.

The three methods had great ability to show all surgical margins in the same section, showing more than $90 \%$ of the surgical margins in all groups. Although statistical differences were not observed, the Figure 10 showed a trend for a better visualization of the surgical margins, when processing separate bisected semicircular samples.

Several factors should be considered when comparing different devices and embedding methods: Easiness of handling, processing speed, quality of histological slides, biologic risk, ergonomics to the histotechnician work, need of some additional apparatus or other source of cryogen, and the final cost should be evaluated $^{5,6}$.

Some features of the method of Rio de Janeiro were not analyzed in this study, however we believe that present some additional advantages when faced with other methods ${ }^{12}$ :

- The ability to process multiple samples simultaneously can bring more agility to the process;

- Easiness of handling the samples outside the restricted space of the cryostat, giving more ergonomic for the Mohs hitotechnician;

- Dry ice has a lower cost when compared to other cryogens agents like liquid nitrogen, and the handling of liquid nitrogen is more dangerous than dry ice ${ }^{12}$.

\section{Conclusion}

Rio de Janeiro technique has a similar effectiveness to the other two techniques analyzed in this article, and it can be an alternative to accelerate the processing of samples in Mohs surgery, maintaining the high quality of the histological slides.

\section{References}

1 - Rogers HW. Is Mohs surgery cost-effective versus traditional surgical excision? Cur Dermatol Rep. 2014;3(2):91-7. doi: 10.1007/ s13671-014-0079-9.

2 - Dim-Jamora KC, Perone JB. Management of cutaneous tumors with Mohs micrographic surgery. Semin Plast Surg. 2008;22(4):247-56. doi: $10.1055 / \mathrm{s}-0028-1095884$.

3 - Otley CC, Salasche SJ. Mohs surgery: efficient and effective. Br J Ophthalmol. 2004;88(9):1228. doi: 10.1136/bjo.2004.047068.

4 - Griffiths RW, Suvarna SK, Stone J. Basal cell carcinoma histological clearance margins: an analysis of 1539 conventionally excised tumours. Wider still and deeper? J Plast Reconstr Aesthet Surg. 2007;60(1): 41-7. doi: 10.1016/j.bjps.2006.06.009.

5 - Lear W, Anderson N, Akeroyd J, Berg, D. A comparison of four Mohs tissue preparation methods using porcine skin. Dermatol Surg. 2010;36:1419-25. doi: 10.1111/j.1524-4725.2010.01651.x.

6- Shoimer I, Warman L, Kurwa HA. Preparation of Mohs micrographic surgery frozen sections: three new pearls leading to a simplified, more-effective process. Dermatol Surg. 2013;39:127982. doi: $10.1111 /$ dsu. 12234 .

7- $\quad$ Nouri K, O'Connell C, Alonso J, Rivas MP, Alonso Y. The Miami Special: a simple tool for quality section mounting in Mohs surgery. J Drugs Dermatol. 2004;3(2):175-7. PMID: 15098973.

8- Hanke CW, Leonard AL, Reed AJ. Rapid preparation of high-quality frozen sections using a membrane and vacuum system embedding machine. Dermatol Surg. 2008;34(1):20-5. doi: 10.1111/j.15244725.2007.34003.x.

9- Bakhtar O1, Close A, Davidson TM, Baird SM Tissue preparation for Mohs frozen sections: a comparison of three techniques. Virchows Arch. 2007;450(5):513-8. doi: 10.1007/s00428-007-0380-0.

10- Silapunt S, Peterson SR, Alcalay J, Goldberg LH. Mohs tissue mapping and processing: a survey study. Dermatol Surg. 2003;29:1109-12. PMID: 14641335.

11- Pike JL, Tusa MG, Lenis A, Butler DF. Face-down cryoembedding of tissue specimens for Mohs Micrographic Surgery Using Stainless Steel Embedding Wells. Dermatol Surg. 2009;35:1545-8. doi: 10.1111/j.1524-4725.2009.01272.x.

12- Sanchez FH, Filho JR, Nouri K, Rizzo LA. Description of a simple method to optimize the process of freezing and embedding tissue in Mohs surgery. Dermatol Surg. 2014;40:472-4. doi: 10.1111/ dsu. 12441 .

13- Davis DA, Pellowski DM, Hanke CW. Preparation of frozen sections. Dermatol Surg. 2004;30:1479-85. doi: 10.1111/j.15244725.2004.30506.x.

14- Wanitphakdeedecha R, Nguyen TH, Minsue Chen TM. In vivo intraoperative relaxing incisions for tissue flattening in Mohs micrographic surgery. Dermatol Surg. 2008;34(8):1085-7. doi: 10.1111/j.1524-4725.2008.34214.x.

15- Ladd S, Cherpelis BS. Scoring of Mohs tissue in one-piece processing to prevent tissue crumbling or detachment from the embedding medium while sectioning. Dermatol Surg. 2009 Oct;35(10):1555-6. doi: 10.1111/j.1524-4725.2009.01274.x.

16- Barlow JO. Using en face inking to define and prevent embedding errors in Mohs specimens. Dermatol Surg. 2011;37:9. doi: 10.1111/j.1524-4725.2011.01982.x.

17- Barlow JO. Using en face ink to guide the conservative sectioning of a Mohs margin. Dermatol Surg. 2011 Dec;37:1730-9. doi:10.1111/ j.1524-4725.2011.02145.x. 
18- Smith-Zangone MJ, Shwartz MR. Frozen sections of skin specimens. Arch Patol Lab Med. 2005;129(12):1536-43. PMID: 16329726.

19- Finley EM. The principles of Mohs micrographic surgery for cutaneous neoplasia. Ochsner J. 2003;5(2):22-33. PMID: 22826680.

\section{Correspondence:}

Frederico Hassin Sanchez

Rua Nascimento Silva, 66/306

22421022 Rio de Janeiro - RJ Brasil

Tel.: (55 21)3852-0102

fredhsanchez@gmail.com

Received: Apr 03, 2016

Review: Jun 08, 2016

Accepted: July 11, 2016

Conflict of interest: none

Financial source: none

${ }^{1}$ Research performed at Laboratory of Experimental Surgery, Department of Science Surgery, Medical School, Universidade Federal do Rio de Janeiro (UFRJ), Brazil. Part of Master degree thesis, Postgraduate Program in Surgical Science. Tutor: Joaquim Ribeiro Filho. 